\title{
Complementarity and quantum walks
}

\author{
Viv Kendon ${ }^{1,2, *}$ and Barry C. Sanders ${ }^{3,4}$ \\ ${ }^{1}$ QOLS, Optics Section, Blackett Laboratory, Imperial College, London, SW7 2BW, United Kingdom \\ ${ }^{2}$ School of Physics and Astronomy, University of Leeds, Leeds, LS2 9JT, United Kingdom \\ ${ }^{3}$ Institute for Quantum Information Science, University of Calgary, Alberta T2N 1N4, Canada \\ ${ }^{4}$ Centre for Quantum Computer Technology, Macquarie University, Sydney, New South Wales 2109, Australia
}

(Received 7 April 2004; published 14 February 2005)

\begin{abstract}
We show that quantum walks interpolate between a coherent "wave walk" and a random walk depending on how strongly the walker's coin state is measured; i.e., the quantum walk exhibits the quintessentially quantum property of complementarity, which is manifested as a tradeoff between knowledge of which path the walker takes vs the sharpness of the interference pattern. A physical implementation of a quantum walk (the quantum quincunx) should thus have an identifiable walker and the capacity to demonstrate the interpolation between wave walk and random walk depending on the strength of measurement.
\end{abstract}

DOI: 10.1103/PhysRevA.71.022307

PACS number(s): 03.67.Lx, 03.65.Ta

\section{INTRODUCTION}

Random walks are essential to physics as stochastic phenomena, to mathematics as Wiener processes, and to computer science for algorithms. The quantum walk, both in its continuous [1] and in its discrete [2-4] incarnations, is receiving significant attention because it is a natural generalization of random walks to quantum systems, because quantum walks may be physically implemented by quincunxes [5-7], and because quantum walks could provide a basis for future quantum algorithms [8-10]. However, an identifiable benefit of the quantum walk, namely the enhancement of spreading over its classical counterpart, is a wave phenomenon which has been realized interferometrically in an optical quincunx [11], and proposed in other settings [12-14]. The realization of aspects of the quantum walk in a classical optics setting has raised the question of what exactly is "quantum" about the quantum walk. We resolve this issue of comparing and contrasting the deterministic wave walk vs a genuine quantum walk by properly accounting for the role of complementarity [15-17].

Although complementarity has been at the heart of quantum mechanics since the dawn of the subject $[16,17]$, studies of complementarity often focus on simple, illustrative cases such as two-slit interference [18] and two-channel interferometry $[19,20]$; we significantly expand the field by providing an analysis of complementarity for general graphs. To incorporate complementarity into quantum walks, we extend from the typical view of a quantum walk defined as unitary local transition rules over the Hilbert space for the vertices of the graph and the states of the walker's coin by allowing a measurement process, either on the transitions, or on the coin outcomes, or both. The measurement is performed by entangling the walker or coin to ancillary degrees of freedom, with the strength of coupling to the ancilla determining whether the quantum walk is coherent (no coupling to ancilla yielding the unitary quantum walk) or random (strong coupling that

\footnotetext{
*Electronic address: V.Kendon@leeds.ac.uk
}

yields full information on the walker's path).

We then define a quantum quincunx as a physical implementation of a quantum walk, which must have an identifiable walker and interpolate between a random walk and a unitary quantum walk as the measurement strength is varied. More precisely, we require a quantum quincunx to have (i) a single walker, (ii) a measurement process that can be employed to acquire varying degrees of knowledge about the path of the walker, and (iii) an identifiable interference phenomenon whose deterioration can be linked to the acquisition of knowledge about the walker's path. This view on complementarity (one walker and a tradeoff between whichpath vs interference) follows the information-theoretic perspective of complementarity introduced by Wootters and Zurek [18].

We proceed as follows: first we provide background on complementarity in Sec. II and then introduce our notation for general graphs, recalling the definition of a classical random walk on such graphs, in Sec. III. This is followed in Sec. IV by the definition in our notation of a unitary quantum walk on a general graph. In Sec. V we extend the definition to include partial measurements of the quantum walker. An example of the walk on a $N$-cycle is given in Sec. VI. In Sec. VII we describe in detail how to perform a measurement of the path taken by the walker by measuring the state of the walker's coin, followed by a general treatment of quantum walks with nonunitary evolutions in Sec. VIII. In Sec. IX we discuss how the wave walk relates to quantum walks, and in Sec. X we summarize our results.

\section{COMPLEMENTARITY FOR QUANTUM WALKS}

In its original formulation $[16,17]$, complementarity is the principle that one classical description of a system, which explains certain phenomena for a quantum system, is incompatible with the simultaneous use of another classical description used to explain other phenomena. In simpler terms, a quantum system can exhibit different, incompatible properties that are each manifested under different circumstances. The most well-known example of complementarity is wave- 
particle duality: objects such as single electrons or single photons can be described as being corpuscular (particlelike) under some circumstances (when the phenomenon being studied, such as particle detection, can be explained by describing these objects as localized, indivisible particles) and undular (wavelike) under other circumstances (when the phenomenon being studied, such as interferometry, can be explained by regarding the objects as extended, interfering waves). The attributes of waves and particles make these two descriptions mutually incompatible, yet electrons or photons can be made to exhibit the features of both these two incompatible descriptions depending on how they are observed.

Complementarity is at the heart of quantum mechanics. Electrons and photons are described by quantum theory in order that these mutually incompatible descriptions can be reconciled. Quantum mechanics provides a unified framework for describing quantum systems that can be corpuscular or wavelike under different circumstances. Systems are in fact regarded as quantum when complementarity is manifest.

Although the early descriptions of complementarity concerned mutually incompatible measurements, Wootters and Zurek presented an information-theoretic description of complementarity, which elucidates that complementarity can be quantified as a tradeoff between knowledge of which way each particle goes vs the sharpness of the interference pattern obtained via repeated preparations and measurements [18]. This tradeoff between corpuscular and undular behavior has been examined in detail for photons both in a theoretical context using a photon number quantum nondemolition measurement [19] and experimentally using nondeterministic linear optical gates [21].

While complementarity has been well studied in quantum physics, its role in identifying the "quantumness" of quantum information tasks has not been explored. Recent controversy over what is "quantum" about quantum walks motivates us to examine the role of complementarity in this context. The controversy over the quantum walk is exemplified by the statement by Knight et al. in the abstract of their paper entitled "Quantum walk on the line as an interference phenomenon" that, "the coined quantum walk on a line can be understood as an interference phenomenon, can be classically implemented, and indeed already has been" [12]. In their conclusions, they state that they have "shown that the [quantum walk] along a line can be simulated in a purely classical implementation, involving nothing more than wave interference of electromagnetic fields." Their work shows that the quantumness of the coin, which is a spin- $-\frac{1}{2}$ particle for the quantum walk on the line, and its possible entanglement with the walker's path, do not by themselves make the quantum walk quantum. The question then arises whether this reasoning is sufficient to claim as they do, that the quantum walk is purely a wave phenomenon that "can be simulated ...[by] wave interference of electromagnetic fields."

Our position is that the quantum walk may indeed be implemented by an optical system, but not by one that is strictly described by classical electromagnetic theory. The optical quincunx of Bouwmeester et al. [11] certainly displays the interference features of the quantum walk on the line, but the quantumness of the quantum walk must connect two seemingly incompatible descriptions: there is a single

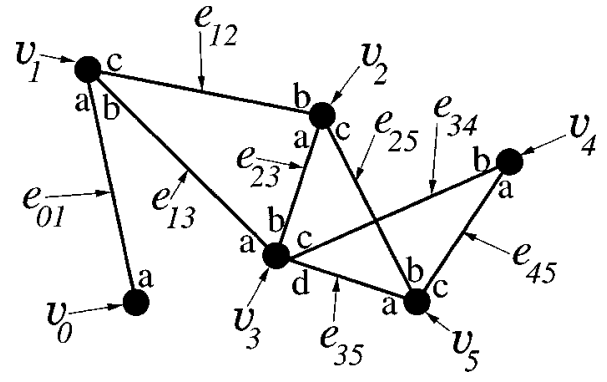

FIG. 1. An example of a general graph, with $N=6$ vertices labeled $v_{0}, v_{1} \cdots v_{5}$, and eight edges labeled $e_{01} \cdots e_{45}$. A labeling of the possible choices of paths from each vertex is given by the letters $\{\mathrm{a}, \mathrm{b}, \mathrm{c}, \mathrm{d}\}$, the degree of this graph being 4 .

walker at a time who can opt for different paths that interfere with each other, and the acquisition of information about the path destroys the interference and restores the classical walk. An experiment that observes one phenomenon of the quantum walk, the interference, is really only observing a "wave walk"; we will show that a quantum optical quincunx can identify that there has been one walker, learn about the path, and show that interference diminishes as path information is obtained.

\section{GENERAL GRAPHS}

We cast our discussion of quantum walks in a very general setting where the walk takes place on a general graph $G(V, E)$ with

$$
V=\left\{v_{j} ; j \in \mathbb{Z}_{N}\right\}
$$

the set of vertices, and $E=\left\{e_{j j^{\prime}}\right\}$ the set of edges, where $e_{j j^{\prime}}$ connects vertices $v_{j}$ and $v_{j^{\prime}}$, as shown in Fig. 1. The number of edges adjoining vertex $v_{j}$ is $d_{j}$ and the degree of the graph is $d=\max _{j} d_{j}$. The complexity of the graph is associated with $|V|=N$, and the degree $d$ is constant as $N$ varies. For simplicity, we assume undirected edges $\left(e_{j j^{\prime}} \equiv e_{j^{\prime} j}\right)$, and at most one edge between any pair of vertices $\left(e_{j j}\right.$ is unique).

In a random walk, the walker's choice of which edge to follow from a given vertex is random (for which we include a $d$-dimensional coin), but this choice of edges can be weighted (some edges are preferred over others) or biased (the coin outcomes are not uniformly distributed over all choices). In general the nature of the coin is correlated with the vertex from which the coin is tossed, e.g., the coin is $d_{j}$-sided at vertex $v_{j}$ or the bias of the coin may be $j$ dependent.

\section{UNITARY QUANTUM WALK}

In contrast to the classical random walk, the quantum walk permits the walker to follow all edges in a superposition state, essentially as Feynman paths through the graph. We first describe the unitary quantum walk, and then generalize to include measurement of the walker's progress. The unitary quantum walk is deterministic, the walker's wave function is in the Hilbert space $\mathcal{H}_{\mathrm{vc}}$ which contains the $\mathrm{N}$-dimensional Hilbert space, 


$$
\mathcal{H}_{\mathrm{v}}=\operatorname{span}\left\{|j\rangle_{\mathrm{v}}: j \in \mathbb{Z}_{N, \mathrm{v}}\left\langle j \mid j^{\prime}\right\rangle_{\mathrm{v}}=\delta_{j j^{\prime}}\right\} \subset \mathcal{H}_{\mathrm{vc}},
$$

of vertex states. For a coin we have a $d$-dimensional Hilbert space,

$$
\left.\mathcal{H}_{\mathrm{c}}=\operatorname{span}\left\{|k\rangle_{\mathrm{c}}: k \in \mathbb{Z}_{d}\right\} \text { and }{ }_{\mathrm{c}}\left\langle k \mid k^{\prime}\right\rangle_{\mathrm{c}}=\delta_{k k^{\prime}}\right\},
$$

where $d$ is the degree of the graph. The basis states of $\mathcal{H}_{\mathrm{vc}}$ are given by

$$
\mathcal{B}_{\mathrm{vc}}=\left\{|j, k\rangle \equiv|j\rangle_{\mathrm{v}}|k\rangle_{\mathrm{c}} ; j \in \mathbb{Z}_{N}, k \in \mathbb{Z}_{d}\right\}
$$

with cardinality $N d$. For a basis state $|j, k\rangle$, the index $j$ identifies the vertex number and $k$ the $k$ th state of the coin. For an edge $e_{j j}$, we associate the coin state $k$ with the edge at $v_{j}$, and the coin state $k^{\prime}$ with the other end of the edge at $v_{j^{\prime}}$. The values of $k$ and $k^{\prime}$ are arbitrary but fixed throughout the quantum walk, to ensure the quantum walker traverses the graph in a consisent manner. We define the mapping

$$
\zeta: Z_{N} \times \mathbb{Z}_{d} \rightarrow \mathbb{Z}_{N} \times \mathbb{Z}_{d}:(j, k) \mapsto \zeta(j, k)=\left(j^{\prime}, k^{\prime}\right),
$$

where $(j, k)$ and $\left(j^{\prime}, k^{\prime}\right)$ label each end of $e_{j j^{\prime}}$.

The quantum walk undergoes a unitary evolution by repetition of two steps: a coin toss and a conditional swap. The coin operator

$$
C: \mathcal{H}_{\mathrm{vc}} \rightarrow \mathcal{H}_{\mathrm{vc}}:|j, k\rangle \mapsto \sum_{\tilde{k} \in Z_{d}} C_{k \hat{k}}^{j}|j, \widetilde{k}\rangle_{\mathrm{c}}
$$

is a block diagonal matrix with each block labeled by $j$. The $j$ dependence of the coin matrix allows sufficient freedom in the quantum walk dynamics for the quantum coherence properties of the coin to vary between vertices, for vertices to act as origins and end points, and for vertices to have different degrees from each other. If $v_{j}$ has degree $d_{j}<d$, we require $C_{k \tilde{k}}^{j}=0$ for all $\tilde{k}$ values not used to label an edge at $v_{j}$. This restricts the coin operator so it only produces states that have a valid mapping under $\zeta$.

If

$$
C_{k \bar{k}}^{j}=C_{k \tilde{k}}^{j^{\prime}} \forall j, j^{\prime},
$$

we have the special case of a fixed degree graph where the coin operator is identical for all vertices. As examples, a two-sided coin has been employed in analyses of the quantum walk on the line [3] and quantum walk on the cycle [4], and a multisided coin for quantum walks on regular lattices in higher dimensions [22,23].

The unitary conditional swap operator is given by

$$
S: \mathcal{H}_{\mathrm{vc}} \rightarrow \mathcal{H}_{\mathrm{vc}}:|j, k\rangle \mapsto\left|j^{\prime}, k^{\prime}\right\rangle,
$$

which updates the position of the walker and the coin state according to the mapping $\zeta$ in Eq. (5), i.e., moves the walker and coin to the vertex $v_{j^{\prime}}$ along edge $e_{j j^{\prime}}$. We note that, by our stipulation that $k$ and $k^{\prime}$ label opposite ends of $e_{j j^{\prime}}$, it follows that $S=S^{-1}$, and is thus unitary as required for quantum evolution. The sequence of a coin flip and a conditional swap is a transition over the unit time step, which we denote by unitary $T=S C$.

This formulation of a (pure state) coined quantum walk on a general graph has appeared in a different form due to
Watrous [2], and is also described by Ambainis [10]. The interferometric scheme of Hillery et al. [24] is also equivalent. The astute reader will have noted that there is thus far nothing random in the dynamics of a unitary quantum walk, it being a perfectly deterministic, reversible unitary evolution. Randomness does arise if one measures the position of the walker after a number of time steps, when the walker will be found on one of the vertices with a probability given by the squared modulus of the walker's wave function over the graph.

\section{NONUNITARY QUANTUM WALK}

We now generalize to include measurements as part of each step of the quantum walk dynamics (rather than only after the final step). Quantum walks with measurements have been considered by Aharonov et al. [25] though with different motivations from ours. The inclusion of measurements requires nonunitary evolution, therefore we introduce density matrices to describe the walker's state. The (time-dependent) density operator

$$
\rho=\sum_{j, k} \sum_{j^{\prime}, k^{\prime}} \rho_{j k}^{j^{\prime} k^{\prime}}|j, k\rangle\left\langle j^{\prime}, k^{\prime}\right|
$$

is a positive ( $\rho=\rho^{\dagger}$ with positive real spectrum), unit-trace, bounded linear operator on $\mathcal{H}_{\mathrm{vc}}$, in the basis $\mathcal{B}_{\mathrm{vc}}$, Eq. (4). The state is pure iff $\rho^{2}=\rho$. A typical initial condition is $\rho(t=0)$ $=|0,0\rangle\langle 0,0|$ corresponding to the walker starting at vertex $v_{0}$ carrying a coin in the 0 state.

In general the density operator is mapped to a new density operator via a completely positive, or CP, map,

$$
\mathcal{T}: \rho \mapsto \mathcal{T} \rho .
$$

The CP map $\mathcal{T}$ performs one coin flip and the conditional swap over one time step. As a CP map,

$$
\mathcal{T} \rho=\sum_{i \in \Theta} T_{i}^{\dagger} \rho T_{i}, \quad \sum_{i \in \Theta} T_{i}^{\dagger} T_{i}=1,
$$

with $i$ an index of nonunitary evolutionary "instances" and $T_{i}$ the corresponding Kraus operator. These instances may be discerned by a measurement record, with $i$ the record index. The cardinality of $\Theta$ can be finite, countably infinite, or even uncountable. In the case of unitary evolution, $\Theta$ has a cardinality of 1 , so there is a unique, unitary $T$ for which $\rho \mapsto \mathcal{T} \rho=T \rho T^{\dagger}$. Unitary quantum walk evolution can thus be expressed as

$$
\rho(t)=\mathcal{T}^{t} \rho(0), \mathcal{T} \equiv \mathcal{S C}, \mathcal{S C} \rho \equiv S C \rho C^{\dagger} S^{\dagger},
$$

where, for the discrete time walk, we assume $t \in \mathbb{Z}$. Thus, for the unitary walk, the transition is given by $T=S C$, and the nonunitary walk can be understood as a collection, or sum, of instances of nonunitary coin flips, with randomness, followed by a conditional swap.

\section{WALK ON THE CYCLE}

At this stage application to a well-known example is helpful, and we consider the quantum walk on a cycle, see Fig. 2. 


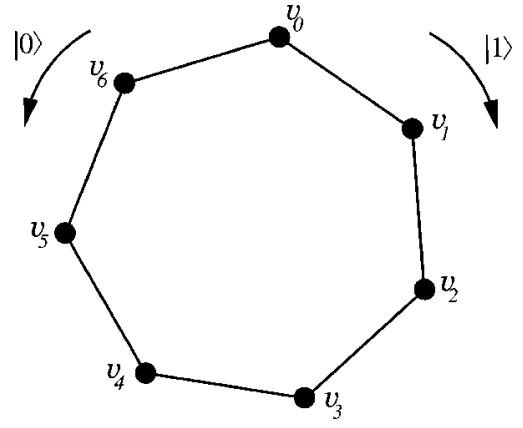

FIG. 2. An example of a cycle graph, with $N=7$ vertices labeled $v_{0}, v_{1} \cdots v_{6}$, and seven edges. Arrows indicate the direction of travel for coin state $|0\rangle$ (anticlockwise) and $|1\rangle$ (clockwise). The cyclic labeling of the vertices does away with the need to label the edges.

The quantum walk on the cycle has the advantages that $\mathcal{H}_{\mathrm{v}}$ is finite-dimensional (as opposed to the walk on the line, for example, which has infinitely many vertices, hence an infinite-dimensional Hilbert space), $d=2$ for all vertices, which is the smallest nontrivial degree, and the quantum walk on the cycle may be experimentally achievable $[5,6]$. The Hilbert space for a two-sided coin is given by $\mathcal{H}_{\mathrm{c}}=\mathcal{H}_{2}$ for

$$
\mathcal{H}_{2}=\operatorname{span}\{|0\rangle,|1\rangle\}
$$

The Hilbert space for the graph + coin is given by

$$
\mathcal{H}_{\mathrm{vc}}=\mathcal{H}_{\mathrm{v}} \otimes \mathcal{H}_{\mathrm{c}} .
$$

We can choose all blocks of the coin matrix to be identical $2 \times 2$ matrices, in this case the unbiased two-sided coin operator is given by a generalized Hadamard transformation [23]

$$
H_{\varphi} \equiv \frac{1}{\sqrt{2}}\left(\begin{array}{cc}
1 & -i e^{\mathrm{i} \varphi} \\
i e^{-\mathrm{i} \varphi} & -1
\end{array}\right)
$$

with a free phase degree of freedom $\varphi$ (typically $\varphi=\pi / 2$ ). Assuming that the vertices are labeled in sequence around the cycle, we can employ the simplicity of assigning $|0\rangle$ to moving from $v_{j}$ to $v_{j+1}$ and $|1\rangle$ to moving from $v_{j}$ to $v_{j-1}$ (rather than labeling each end of the edges). The conditional swap operation becomes

$$
S=\sum_{\varepsilon \in\{0,1\}} \sum_{j \in Z_{N}}\left|j-(-1)^{\varepsilon} \bmod N\right\rangle\langle j|\otimes| \varepsilon\rangle\langle\varepsilon|
$$

yielding the transformation $S|j, \varepsilon\rangle=\left|j-(-1)^{\varepsilon}, \varepsilon\right\rangle$.

The quantum walk on the cycle has been well studied: it mixes faster than a classical random walk, both for unitary quantum walks [4], and when a small amount of decoherence is applied in the form of nonunitary random measurements of the coin and/or the walker's position [26].

\section{MEASUREMENTS OF THE COIN}

Now that we have the machinery in place to describe nonunitary quantum walks on general graphs, we can return to the question of complementarity and consider how to track the path of the walker. We will explain this in detail for a two-sided coin such as the one used for the walk on a cycle described in the previous section. Suppose that measurements of the coin state are performed after each coin flip, in the coin state basis $\{|0\rangle,|1\rangle\}$. This measurement can be performed by adding an ancilla that serves as the coin meter, and the meter state becomes correlated by interacting with the coin. The Hilbert space for the ancilla is of the same dimension as the Hilbert space for the coin, so the meter's Hilbert space is $\mathcal{H}_{\mathrm{m}}=\mathcal{H}_{2}$, given by Eq. (13). Letting

$$
\begin{gathered}
\sigma_{+} \equiv|1\rangle_{\mathrm{c}}\left\langle 0\left|=\sigma_{-}^{\dagger}, \tau_{+} \equiv\right| 1\right\rangle_{\mathrm{m}}\langle 0|=\tau_{-}^{\dagger}, \\
2 \sigma_{x}=\sigma_{+}+\sigma_{-}, 2 \mathrm{i} \sigma_{y}=\sigma_{-}-\sigma_{+}, \\
2 \sigma_{z}=\left[\sigma_{+}, \sigma_{-}\right], 2 \tau_{z}=\left[\tau_{+}, \tau_{-}\right],
\end{gathered}
$$

the meter couples to the coin via the interaction [27]

$$
\begin{aligned}
W(\beta)= & \left(e^{\mathrm{i}(\pi / 4) \sigma_{y}} \otimes e^{-\mathrm{i}(\pi / 4) \tau_{x}}\right) e^{-\mathrm{i} \beta(\pi / 4) \sigma_{x} \otimes \tau_{x}}\left(e^{-\mathrm{i}(\pi / 4) \sigma_{y}}\right. \\
& \left.\otimes e^{-\mathrm{i}(\pi / 4) \tau_{z}}\right)\left(1 \otimes e^{-\mathrm{i} \pi(1-\beta) \tau_{y} / 4}\right)\left(1 \otimes e^{-\mathrm{i} \pi \tau_{z} / 4}\right) .
\end{aligned}
$$

The uncoupled case corresponds to $W(0)$, and $W(1)$ corresponds to the strong-coupling limit (a controlled-NOT operation) with resultant sharp measurements. The interpolation between the limits is achieved by allowing $\beta$ to vary continuously over the domain $[0,1]$.

To perform a measurement, we first prepare the meter in the $|0\rangle_{\mathrm{m}}$ state, then allow it to interact with the coin, which is in an arbitrary qubit state $\gamma|0\rangle_{\mathrm{c}}+\eta|1\rangle_{\mathrm{c}}$,

$$
\begin{aligned}
W(\beta)\left(\gamma|0\rangle_{\mathrm{c}}+\eta|1\rangle_{\mathrm{c}}\right)|0\rangle_{\mathrm{m}}= & \gamma|00\rangle_{\mathrm{cm}}+\eta|1\rangle_{\mathrm{c}}\left[\cos (\beta \pi / 2)|0\rangle_{\mathrm{m}}\right. \\
& \left.+\sin (\beta \pi / 2)|1\rangle_{\mathrm{m}}\right] .
\end{aligned}
$$

Tracing over the meter state yields the $2 \times 2$ coin densitymatrix transformation

$$
\begin{gathered}
\operatorname{Tr}_{\mathrm{m}}\left\{W(\beta)\left[\left(\begin{array}{cc}
|\gamma|^{2} & \gamma \eta^{*} \\
\gamma^{*} \eta & |\eta|^{2}
\end{array}\right) \otimes\left(\begin{array}{ll}
1 & 0 \\
0 & 0
\end{array}\right)\right] W^{\dagger}(\beta)\right\} \\
=\left(\begin{array}{cc}
|\gamma|^{2} & \gamma \eta^{*} \cos (\beta \pi / 2) \\
\gamma^{*} \eta \cos (\beta \pi / 2) & |\eta|^{2}
\end{array}\right) .
\end{gathered}
$$

The coin values correspond to the pointer basis $[28,29]$, with the degree of decoherence depending on $\beta$.

These measurements of the coin provide information on path choices that the walker makes. If the walker's initial state is localized at one known vertex, then perfect coin state measurements suffice to inform the observer as to the exact trajectory of the walker. The parameter $\beta$ allows the observer to acquire as much or as little information as desired, thereby allowing the degree of knowledge of which trajectory the walker followed and the complementary degree of coherence reduction to be controlled. To see the effect of varying $\beta$, first note that $\beta=0$ corresponds to no measurement of the coin. In this case the last matrix in Eq. (22) corresponds to perfect coherence because the $\cos (\beta \pi / 2)$ term becomes unity. As $\beta$ increases from 0 to $1, \cos (\beta \pi / 2) \rightarrow 0$ and perfect decoherence emerges: the random walk arises because the end states of the quantum walk are just probability-weighted sums of each path. 


\section{QUANTUM WALKS IN OPEN SYSTEMS}

Nonunitary evolution of the quantum walk of course can also arise from other processes besides measuring the coin; in the case of the quantum walk on the cycle in a cavity quantum electrodynamics realization, cavity damping is naturally associated with measuring the phase of the intracavity field [6], which corresponds to direct measurements of the vertex states for the quantum walk on the cycle. In this case the decoherence mechanism is due to measurements of vertex occupation, not the coin state, but the classical random walk emerges all the same.

For more general quantum walks, weak or strong measurements of the state of the coin generalize the unitary evolution of Eq. (12) to the nonunitary case

$$
\mathcal{T}: \rho \mapsto \sum_{i \in \Theta} S C_{i} \rho C_{i}^{\dagger} S^{\dagger}
$$

with $C_{i}$ the Kraus operators for different instances of coinstate randomization.

According to Naimark's theorem [30], the positive operator-valued measure of coin state that yields the CP map of Eq. (23) can be realized by coupling the coin state in $\mathcal{H}_{\mathrm{c}}$ to an ancilla in an extended Hilbert space $\mathcal{H}_{\mathrm{a}}$ and performing projective (von Neumann) measurements of the ancilla state. The positive operator-valued measure of the coin state is then obtained by tracing over ancilla states. The coin can be considered as a qudit of dimension $d$, and a $d$-dimensional ancillary qudit suffices. The qudit-qudit coupling that interpolates from no measurement of the coin state to weak measurements to sharp measurements with full information is a complicated generalization [31] of the qubit-qubit coupling in Eq. (20). A projective measurement of the ancilla gives none, some, or all of the information about the coin qudit, depending on the qudit-qudit coupling strength, which, with full measurements, then yields the classical walk on the graph. Measurements of coin states are sufficient to reduce a quantum walk on the graph to a classical walk on the same graph provided the walker starts in a basis state [32].

\section{OPTICAL QUANTUM QUINCUNX}

Having incorporated measurement into quantum walks and shown that measurement introduces complementarity, which interpolates between the random walk and the unitary quantum walk, we now consider the quantum quincunx: a physical system which implements a quantum walk. Our requirements for a quantum quincunx are that the system (i) has an identifiable walker, (ii) exhibits both the unitary quantum walk and the random walk as complementary features of the quantum walk, and (iii) interpolates between these two complementary extremes according to a controllable degree of measurement that provides information about the walker's path.

The quantum quincunx can be realized in various physical systems [5-7], but here the optical quincunx provides a convenient system for understanding quincunxes and the requirements for a quantum quincunx. Furthermore, the optical quincunx has been realized experimentally as an interferom- eter [11]. The typical source for interferometry is a coherent laser source, which is often described as producing a coherent state [14], a certain coherent superposition of different numbers of photons, where the photon number states are given by $|n\rangle$, and the indeterminacy of the photon number is necessary in order for the phase variance to be small. Although interferometric experiments can be fully described by classical fields, the coherent state provides a bridge to connect the quantum and classical field descriptions, with the photons playing the role of the walkers in the system.

Second quantization seems to present a dilemma with respect to the requirement of an identifiable walker: a coherent state of walkers is given by

$$
|\alpha\rangle_{\mathrm{w}}=e^{-|\alpha|^{2} / 2} \sum_{n=0}^{\infty} \frac{\alpha^{n}}{\sqrt{n !}}|n\rangle_{\mathrm{w}},
$$

where $|n\rangle_{\mathrm{w}}$ is a number state of walkers along the graph. The parameter $\alpha$ is complex, whose square modulus $|\alpha|^{2}$ is the mean energy, the discrete energy distribution is Poissonian, and $\arg (\alpha)$ is the phase. Let us deal with two challenges: (a) the quantum walk with multiple walkers and (b) the indefiniteness of the number of walkers. With respect to challenge (a), generalizing the quantum walk from one to $n$ walkers is straightforward: as the $n$-walker system involves noninteracting walkers, so the Hilbert space for the walkers is given by $\mathcal{H}_{\mathrm{vc}}^{\otimes n}$, and the completely positive map generalizes to $\mathcal{T}^{\otimes n}$. The $n$-walker system is a simple extension of the one-walker system as a tensor product of $n$ one-walker systems with one time step given by $\mathcal{T}^{\otimes n} \rho^{\otimes n}$. Each walker carries its own coin, and these $n$ coins are coupled to $n$ meter qudits, so measurement and complementarity arise via this coupling. Essentially this $n$-walker system is equivalent to repeating the onewalker quantum walk $n$ times.

With respect to challenge (b), the wave walk appears to emerge through second quantization of walker number, and the indeterminacy of walker number in Eq. (24) enables the phase, which is complementary to number, to be reasonably sharp in order to provide strong coherence. However, it has become abundantly clear recently [33-35] that the coherent state and number state offer complementary yet convenient alternative representations. The quantum optics implementation [11-14] can be described within the photon number superselection framework [35], so each run of the optical quincunx experiment can be interpreted as having a fixed number of photons, and this number of photons can be postselected by an ideal photon counting measurement on all the output fields.

Complementarity in the quantum walk would be manifested by allowing each photon to be tracked during its evolution. This requirement is not easily met, but a practical approach is as follows: the parameter $|\alpha|^{2}$ in Eq. (24) corresponds to the laser flux, and attenuating the laser so that $|\alpha|^{2} \ll 1$ ensures that multiphoton contributions are negligible. Then each run overwhelmingly corresponds to no photon or one photon. In this single-photon regime [36,37], the presence of the photon can be ascertained by a photodetection at the output: the presence of the photon is postselected when the photodetector at the output clicks, announcing that 
this run of the quantum quincunx had a walker. The photon's path is ascertained by quantum nondemolition measurements, either by a nonlinear optical medium for deterministic detection of the photon without destroying it [19] or by nondeterministic linear optical detection [21]. Operating in a low photon flux regime and performing photon number quantum nondemolition measurements of path would allow a continuous interpolation between the the unitary quantum walk and the random walk, thus extending the optical quincunx to a fully functioning quantum quincunx. We are thus able to elucidate what has been achieved by the optical quincunx of Bouwmeester et al. [11] towards an optical implementation of the quantum walk and what needs to be added to provide a full implementation.

The optical quincunx emulates the undular properties, or interference, of the quantum walk, as stated by Knight et al. [13], but this is performed without an identifiable, single walker. In fact the transition to the distribution for a classical random walk should also be achievable if the interferometer is allowed to decohere. If the relative phases between interferometer paths are fully decohered, for example by dispersive media in the paths, the resultant interferometer output will not correspond to a superposition of paths but rather to an incoherent, probability-weighted distribution of outputs. This incoherent sum of paths is precisely the random walk distribution.

Thus the experiment of Bouwmeester et al. could be modified to demonstrate features of both the random walk distribution and the quantum walk interference effect, but complementarity dictates three criteria to achieve quantumness. Just demonstrating a decoherence of wavelike interference is insufficient to establish the corpuscular property of the objects; one must demonstrate their indivisibility. The walker should be a single photon, and there are several possible methods to achieve this [37]. One is to produce photon pairs, for example via parametric downconversion, and obtain one photon conditioned on detecting its partner; another approach is to produce single photons on demand by a source such as a quantum dot in a strong cavity; a third approach, which is currently the easiest, is to postselect the results on detecting a single photon, thereby eliminating vacuum and multiphoton contributions and sorting data based on one and only one photon having been in the system.

In summary, an optical quincunx can implement the quantum walk when it operates in the single photon regime. In this case the device is a quantum optical quincunx. The quantumness of the quincunx is essential to manifest the complementarity properties of the quantum walk, namely the tradeoff between information about the walker's path and the interference.

\section{CONCLUSIONS}

In this paper we have incorporated complementarity into the theory of quantum walks, thereby addressing the issue of what is "quantum" about the quantum walk, as well as extending the concept of complementarity well beyond the usual physical systems (e.g., interferometry) to quantum walks on general graphs. Our analysis of complementarity in quantum walks builds on the approach of coined quantum walks and replaces unitary evolution by the much more general completely positive map approach, which is relevant to considerations of experimental realizations of quantum walks. Through measurement, the quantum walk may exhibit the unitary quantum walk, the random walk, and intermediate processes depending on the strength of the measurement. We define a quantum quincunx as a physical implementation of a quantum walk, including measurement of path, that can demonstrate the essential properties of complementarity in a quantum walk: interference traded off with which-path information, and the indivisibility of the quantum walker. An extension of the optical quincunx experiment of Bouwmeester et al. [11] operated in the single photon regime with postselection for the presence of a photon, and in which the interferometer arms contained photon nondemolition measurement devices, would turn this into a fully quantum quincunx.

\section{ACKNOWLEDGMENTS}

V.K. appreciates useful discussions with P. L. Knight, E. Roldán, and J. Sipe. B.C.S. acknowledges valuable discussions with S. D. Bartlett, D. W. Berry, M. Hillery, D. Meyer, and J. Watrous. This work was funded in part by UK Engineering and Physical Sciences Research Council Grant No. GR/N2507701, Alberta's informatics Circle of Research Excellence (iCORE), and the Australian DEST Innovation Access Program fund to support collaboration with the European Fifth Framework project QUPRODIS.
[1] E. Farhi and S. Gutmann, Phys. Rev. A 58, 915 (1998).

[2] J. Watrous, J. Comput. Syst. Sci. 62, 376 (2001).

[3] A. Ambainis, E. Bach, A. Nayak, A. Vishwanath, and J. Watrous, in Proceedings of the 33rd Annual ACM Symposium on Theory of Computing (STOC 2001) (Association for Computing Machinery, New York, 2001), pp. 60-69.

[4] D. Aharanov, A. Ambainis, J. Kempe, and U. Vazirani, in Proceedings of the 33rd Annual ACM Symposium on Theory of Computing (STOC 2001) (Ref. [3]), pp. 50-59.

[5] B. C. Travaglione and G. J. Milburn, Phys. Rev. A 65, 032310 (2002).
[6] B. C. Sanders, S. D. Bartlett, B. Tregenna, and P. L. Knight, Phys. Rev. A 67, 042305 (2003).

[7] W. Dür, R. Raussendorf, V. M. Kendon, and H.-J. Briegel, Phys. Rev. A 66, 052319 (2002).

[8] A. M. Childs, R. Cleve, E. Deotto, E. Farhi, S. Gutmann, and D. A. Spielman, in Proceedings of the 35th Annual ACM Symposium on Theory of Computing (STOC 2003) (Association for Computing Machinery, New York, 2003), pp. 59-68.

[9] N. Shenvi, J. Kempe, and K. Birgitta Whaley, Phys. Rev. A 67, 052307 (2003).

[10] A. Ambainis, Int. J. Quantum Inf. 1, 507 (2003). 
[11] D. Bouwmeester, I. Marzoli, G. P. Karman, W. Schleich, and J. P. Woerdman, Phys. Rev. A 61, 013410 (1999).

[12] P. L. Knight, E. Roldán, and J. E. Sipe, Phys. Rev. A 68 , 020301(R) (2003).

[13] P. L. Knight, E. Roldán, and J. E. Sipe, Opt. Commun. 227, 147 (2003)

[14] H. Jeong, M. Paternostro, and M. S. Kim, Phys. Rev. A 69, 012310 (2004).

[15] N. Bohr, Science 111, 51 (1950) [reprinted from Dialectica 2, 312 (1948)].

[16] N. Bohr, Naturwiss. 16, 245 (1928).

[17] N. Bohr, Nature (London) 121, 580 (1928).

[18] W. K. Wootters and W. H. Zurek, Phys. Rev. D 19, 473 (1979).

[19] B. C. Sanders and G. J. Milburn, Phys. Rev. A 39, 694 (1989).

[20] B.-G. Englert, Phys. Rev. Lett. 77, 2154 (1996).

[21] G. J. Pryde, J. L. O'Brien, A. G. White, S. D. Bartlett, and T. C. Ralph, Phys. Rev. Lett. 92, 190402 (2004).

[22] C. Moore and A. Russell, in Proceedings of the 6th International Workshop on Randomization and Approximation Techniques in Computer Science (RANDOM '02), edited by J. D. P. Rolim and S. Vadhan (Springer, New York, 2002), pp. 164178.
[23] T. D. Mackay, S. D. Bartlett, L. T. Stephenson, and B. C. Sanders, J. Phys. A 35, 2745 (2002).

[24] M. Hillery, J. Bergou, and E. Feldman, Phys. Rev. A 68, 032314 (2003).

[25] Y. Aharonov, L. Davidovich, and N. Zagury, Phys. Rev. A 48, 1687 (1992).

[26] V. Kendon and B. Tregenna, Phys. Rev. A 67, 042315 (2003).

[27] D. W. Berry (private communication).

[28] W. H. Zurek, Phys. Rev. D 24, 1516 (1981).

[29] W. H. Zurek, Phys. Rev. D 26, 1862 (1982).

[30] M. A. Nielsen and I. J. Chuang, Quantum Computation and Quantum Information (Cambridge University Press, Cambridge, UK, 2000).

[31] D. Gottesman, Lect. Notes Comput. Sci. 1509, 302 (1999).

[32] C. C. Lopéz and J. P. Paz, Phys. Rev. A 68, 052305 (2003).

[33] K. Mølmer, Phys. Rev. A 55, 3195 (1997).

[34] T. Rudolph and B. C. Sanders, Phys. Rev. Lett. 87, 077903 (2001).

[35] B. C. Sanders, S. D. Bartlett, T. Rudolph, and P. L. Knight, Phys. Rev. A 68, 042329 (2003).

[36] P. Pfleegor and L. Mandel, Phys. Rev. 159, 1084 (1967).

[37] P. Hariharan and B. C. Sanders, Prog. Opt. 36, 49 (1996). 